\title{
Brownian Motion of Charged Particles in a Bath Responding to an External Magnetic Field
}

\author{
V. Lis Ý ${ }^{a, b, *}$ AND J. TÓTHOVÁ ${ }^{a}$ \\ ${ }^{a}$ Department of Physics, Faculty of Electrical Engineering and Informatics, Technical University of Košice, Park \\ Komenského 2, 04200 Košice, Slovak Republic \\ ${ }^{b}$ Laboratory of Radiation Biology, Joint Institute for Nuclear Research, 141980 Dubna, Moscow Region, Russia
}

\begin{abstract}
We consider the Brownian motion in a constant magnetic field. Unlike in the previous theories, both the Brownian particle and the surrounding bath particles, if they are charged, respond to the external field. The bath particles are regarded as harmonic oscillators interacting with the Brownian particle. The derived equations of motion for the Brownian particle have a form of generalized Langevin equations. For the motion in the plane perpendicular to the field, two coupled equations for the Brownian particle velocity projections are obtained. Under the condition of stationarity, the velocity correlation functions are determined by the memory functions of the system. The second fluctuation-dissipation theorem is proved. It has the familiar form, but here the thermal noise depends on the magnetic field.
\end{abstract}

DOI: 10.12693/APhysPolA.137.657

PACS/topics: Brownian motion, magnetic field, bath particles response, generalized Langevin equations, fluctuation-dissipation theorem

\section{Introduction}

The Brownian motion of particles is effectively described by the stochastic Langevin equations of motion which, along with external forces, contain forces originating from random impacts of surrounding molecules. The case where the time scale of the molecular motion is not very much shorter than that of the Brownian particle (BP) corresponds to the generalized Langevin equation (GLE). For this case Kubo [1] derived the so-called second fluctuation-dissipation theorem (FDT). He assumed that the bath particles surrounding the BP are unaffected by the external field. Recently, it was discussed in [2] that such an approach is not realistic and there are a number of physical problems, where not only the tagged BP but also the particles are subjected to the oscillating electric field. In Ref. [3] it has been shown by computer simulations that if the dynamics of a methane molecule solvated in water and subjected to a harmonic potential is modeled by the GLE, the memory function and the thermal noise due to the FDT driving the methane molecule are necessarily affected by the external field. In Ref. [4] we have abandoned the Kubo assumption [1] that the external force does not affect the random force in the GLE and derived the second FDT. By modifying the CaldeiraLegget particle-bath model [5] we found a new form of the GLE in the case when both the BP and the bath particle dynamics are affected by the external confinement potential. In such GLE the memory function explicitly depends on the elasticity constant of the potential.

*corresponding author; e-mail: vladimir.lisy@tuke.sk
In the present contribution, we develop the theory in which the observed BP and the surrounding bath particles both can respond to the external magnetic field. The charged bath particles are regarded as harmonic oscillators interacting with the BP. Equations of motion are derived for the BP. Along the vector of magnetic induction, where there is no effect of the field on the particles' motion, the same GLE is obtained as it follows from the theory $[5,6]$. For the motion across the field, two coupled stochastic equations are obtained for the particle velocity projections on the plane perpendicular to the field. The memory effect of the frictional forces is determined by the coupling between the particle and the bath oscillators and depends on the distribution of the oscillators' natural and cyclotron frequencies. The stochastic forces are, in general, coloured. Under the condition of stationarity, we derive the velocity correlation functions (VCFs) of the BP. Finally, the second FDT is proved. It has the familiar form [1], but now the memory function depends on the magnetic field. As an example, we choose the Drude spectrum [5] for bath frequencies to describe the memory effects in the dynamics of the BP.

\section{Model for the Brownian motion in a magnetic field}

We consider a system containing a tagged particle and a bath of small charged oscillators. The particle is coupled to the oscillators that are not coupled to each other. This coupling is a function of the frequency of the oscillators. Within the linear approximation and in the absence of the magnetic field, the equations of motion for the bath particles and the BP with the positions $\boldsymbol{r}_{i}=\left(x_{i}, y_{i}, z_{i}\right)$ and $\boldsymbol{r}=(x, y, z)$, and momenta $\boldsymbol{p}_{i}=\left(p_{x i}, p_{y i}, p_{z i}\right)$ and $\boldsymbol{p}=\left(p_{x}, p_{y}, p_{z}\right)$, respectively, have 
been derived by Zwanzig [6]. In this theory $[5,6]$, the system is placed in a harmonic potential. Here we assume that the system is in the constant external magnetic field $\boldsymbol{B}$ oriented along the axis $z$. The bath particles of masses $m_{i}$ carry charges $q_{i}$ and the charge of the BP of mass $m$ is $Q$. The equations of motion for such a system of particles are as follows:

$$
\begin{aligned}
& \dot{\boldsymbol{r}}=\frac{\boldsymbol{p}}{m}, \quad \dot{\boldsymbol{p}}=\sum_{i} m_{i} c_{i}\left(\boldsymbol{r}_{i}-\frac{c_{i}}{\omega_{i}^{2}} \boldsymbol{r}\right)+Q \dot{\boldsymbol{r}} \times \boldsymbol{B}, \\
& \dot{\boldsymbol{r}}_{i}=\frac{\boldsymbol{p}_{i}}{m_{i}}, \quad \dot{\boldsymbol{p}}_{i}=-m_{i} \omega_{i}^{2} \boldsymbol{r}_{i}+m_{i} c_{i} \boldsymbol{r}+q_{i} \dot{\boldsymbol{r}}_{i} \times \boldsymbol{B} .
\end{aligned}
$$

Here $c_{i}$ are strengths of coupling between the BP and the oscillators with eigenfrequencies $\omega_{i}$. Along the $z$ axis the motion of the particles is not affected by the magnetic field and will be not considered here. The corresponding GLE for a free BP is well known and studied in a number of works.

To get the equations of motion for the $\mathrm{BP}$ in the plane perpendicular to the field, one has to solve the equations for the bath particle momenta $p_{x i}, p_{y i}$ and substitute the solutions $x_{i}, y_{i}$ into the equations for the momenta $p_{x}$ and $p_{y}$. This can be effectively done by using the Laplace transform (LT) of the time-dependent quantities, e.g., $\tilde{x}(s)=\int_{0}^{\infty} \mathrm{d} t \mathrm{e}^{-s t} x(t)$. So, if $\tilde{y}_{i}(s)$ obtained from (2) is substituted by (1), one gets the uncoupled equation for $\tilde{x}_{i}(s)$ and $\tilde{y}_{i}(s)$,

$$
\begin{aligned}
& \left(s^{2}+\omega_{i}^{2}+\frac{s^{2} \Omega_{i}^{2}}{s^{2}+\omega_{i}^{2}}\right) \tilde{x}_{i}(s)=\left(s+\frac{s \Omega_{i}^{2}}{s^{2}+\omega_{i}^{2}}\right) x_{i}(0) \\
& \quad+\dot{x}_{i}(0)-\frac{\omega_{i}^{2} \Omega_{i}}{s^{2}+\omega_{i}^{2}} y_{i}(0)+\frac{s \Omega_{i}}{s^{2}+\omega_{i}^{2}} \dot{y}_{i}(0) \\
& \quad+c_{i} \tilde{x}(s)+\frac{s c_{i} \Omega_{i}}{s^{2}+\omega_{i}^{2}} \tilde{y}(s)
\end{aligned}
$$

A similar equation for $\tilde{y}_{i}(s)$ is obtained if all $x$ are replaced by $y$ and vice versa, and the cyclotron frequency $\Omega_{i}=q_{i} B / m_{i}$ is changed to $-\Omega_{i}$. The equations contain initial positions of the oscillators, $x_{i}(0), y_{i}(0)$, and their initial velocities, $\dot{x}_{i}(0), \dot{y}_{i}(0)$, all of which are random quantities with zero mean. Now we substitute the solutions for $\tilde{x}_{i}(s)$ and $\tilde{y}_{i}(s)$ in the LT of (1) and then return to the time domain. The final equations for the BP have the form

$$
\begin{aligned}
& m \dot{v}_{x}(t)=Q B v_{y}(t)-\int_{0}^{t} v_{x}\left(t^{\prime}\right) G\left(t-t^{\prime}\right) \mathrm{d} t^{\prime} \\
& +\int_{0}^{t} v_{y}\left(t^{\prime}\right) H\left(t-t^{\prime}\right) \mathrm{d} t^{\prime}+f_{x}(t) .
\end{aligned}
$$

A similar equation holds for $v_{y}(t)$ if $x$ is changed to $y$, $Q$ to $-Q$, and $H$ to $-H$. Here, $f_{x}(t)$ and $f_{y}(t)$ are independent zero-mean projections of the random force. It is seen that Eqs. (4) are of the GLE type but with two functions, $G(t)$ and $H(t)$, determining the memory effects in the BP dynamics. Denoting $\gamma_{i}=\left(\Omega_{i}^{2}+4 \omega_{i}^{2}\right)^{1 / 2}$ and $\gamma_{i}^{ \pm}=\gamma_{i} \pm \Omega_{i}, G(t)$ is expressed as

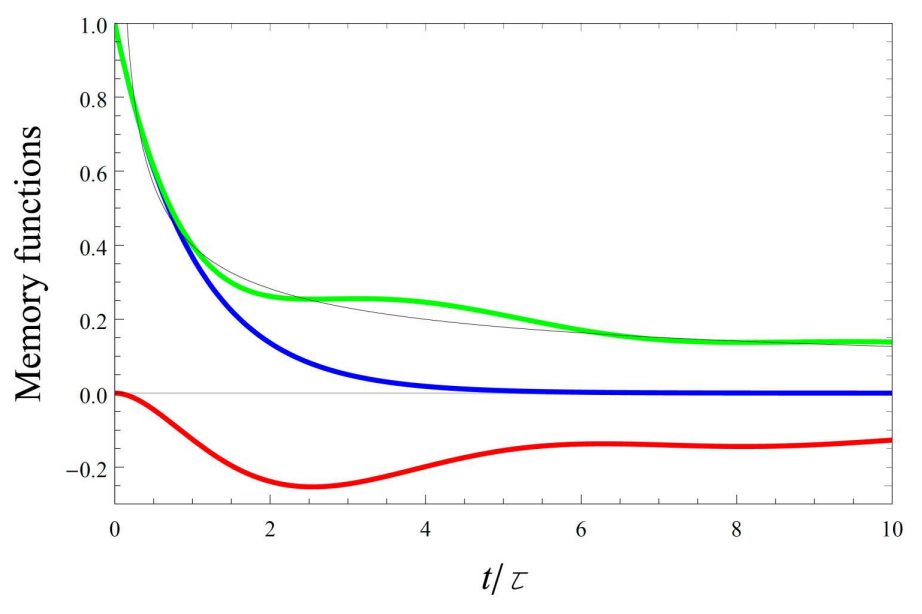

Fig. 1. Time dependence of the memory functions normalized to $\xi / \tau$ for $q B \tau / \mu=1$ and $q>0$. Blue and green lines correspond to $G(t)$ in the absence and presence of bath response to the magnetic field, respectively. The latter case is well described by the asymptote $\sim t^{-1 / 2}$. $H(t)$ is represented by the red line.

$$
G(t)=2 \sum_{i=1}^{N} \frac{m_{i} c_{i}^{2}}{\gamma_{i}}\left[\frac{\cos \left(\gamma_{i}^{+} t / 2\right)}{\gamma_{i}^{+}}+\frac{\cos \left(\gamma_{i}^{-} t / 2\right)}{\gamma_{i}^{-}}\right]
$$

and in the equation for $H(t) \cos (\ldots)$ are just replaced by $\sin (\ldots)$ with the second term in $[\ldots]$ having the sign -. For the correlation functions describing the random motion of the BPs (such as the VCF $C_{v v}(t)=\langle v(0) v(t)\rangle$ ) some general results can be obtained assuming that the system is conditioned to be stationary. So, let us multiply Eq. (4) and a similar equation for $v_{y}(t)$ by $v_{x}(0)$ and $v_{y}(0)$, respectively, and take the statistical average. From the obtained equations for $C_{v_{\alpha} v_{\alpha}}(t)(\alpha=x, y)$, $C_{v_{x} v_{y}}(t)=\left\langle v_{x}(t) v_{y}(0)\right\rangle$, and $C_{v_{y} v_{x}}(t)=\left\langle v_{y}(t) v_{x}(0)\right\rangle$ with $C_{v_{x} v_{y}}(0)=0$, we again turn to the LT. With the commonly used causality principle due to which $\left\langle v_{\alpha}(0) f_{\alpha}(t)\right\rangle=0$ for $t>0$, the convolution theorem, and equipartition, $C_{v_{\alpha} v_{\alpha}}(0)=k_{\mathrm{B}} T / m$, we find the VCF

$$
\tilde{C}_{v_{\alpha} v_{\alpha}}(s)=k_{\mathrm{B}} T \frac{m s+\tilde{G}(s)}{[m s+\tilde{G}(s)]^{2}+[Q B+\tilde{H}(s)]^{2}} .
$$

The expression for $\tilde{C}_{v_{x} v_{y}}(s)=-\tilde{C}_{v_{y} v_{x}}(s)$ differs only by the numerator, which is $Q B+\tilde{H}(s)$. These functions are related to all other relevant correlation functions, such as the positional autocorrelation function $C_{x x}(t)=\langle x(0) x(t)\rangle$, the mean square displacement (MSD) $X(t)=2\left[C_{x x}(0)-C_{x x}(t)\right]$, or the time-dependent diffusion coefficient $D_{x}(t)=\dot{X}(t) / 2$. In the LT, $\tilde{X}(s)=$ $2 \tilde{D}_{x}(s) / s=2 \tilde{C}_{v_{x} v_{x}}(s) / s^{2}$. Analogous relations hold for the $y$ direction.

Moreover, if we multiply Eq. (4) by $f_{x}(0)=m \dot{v}_{x}(0)-$ $Q B v_{y}(0)$ and take the average, the time correlation functions of the random forces, $C_{f_{x} f_{x}}(t)=\left\langle f_{x}(t) f_{x}(0)\right\rangle$ can be expressed through the already derived VCFs. Repeating the same for the $y$ component of the velocity with 
$f_{y}(0)=m \dot{v}_{y}(0)+Q B v_{x}(0)$ and by using the identities for stationary VCFs $[1,4]$, we come to the important result

$$
\tilde{C}_{f_{\alpha} f_{\alpha}}(s)=k_{\mathrm{B}} T \tilde{G}(s), \quad \alpha=x, y .
$$

This is a generalization of the Kubo second FDT, which thus holds also in the case when the bath responses to the external magnetic field. The role of the memory function is played only by $G(t)$. When the bath response is ignored, $\Omega_{i}=0, H(t)=0$, and Eqs. (4)-(7) coincide with the previously derived equations for the BM of particles in a magnetic field [7].

There are many possibilities to specify the obtained results for a concrete spectral distribution of the bath oscillators' frequencies. We have done it for the Drude spectrum [5], assuming that all particles have the same mass $\mu$ and charge $q$. The relaxation time of the thermal force is $\tau$ and the friction coefficient of the BP is $\xi$ when $\tau \rightarrow 0$. An illustration of the numerical calculations of the memory functions dependence on time is shown in Fig. 1. Analytical results for $G(t)$ and $H(t)$ have also been obtained. This analysis and the found correlation functions of interest will be published elsewhere. We only note that for $q \neq 0$ the MSDs in the $x$ and $y$ directions are subdiffusive: $X(t)=Y(t) \approx 2 D_{1 / 2} t^{1 / 2}$ as $t \rightarrow \infty$, with the fractional diffusion coefficient $D_{1 / 2}=\left(k_{\mathrm{B}} T / \xi\right) \sqrt{2 \mu / \pi|q| B}$.

\section{Conclusions}

Unlike from the previous works on the Brownian motion in external magnetic fields, in the presented theory, the response of the surrounding bath particles is also taken into account. This leads to several new results.
First, although we have proved that the Kubo second FDT remains valid, the thermal random force and the memory functions of the derived generalized Langevin equations now depend on the magnetic induction $B$. Explicit expressions for these functions have been obtained. The general formulae found possess large possibilities for further developments. This concerns, e.g. the use of the frequency distribution of the bath oscillators, which can be very different for real systems. As an example, we considered the popular Drude spectrum, which at long times leads to sub-diffusion with the fractional parameter $1 / 2$.

\section{Acknowledgments}

This work was supported by the SGA grant of the Slovak Republic No. 1/0250/18.

\section{References}

[1] R. Kubo, Rep. Prog. Theor. Phys. 29, 255 (1966).

[2] B. Cui, A. Zaccone, Phys. Rev. E 97, 060102(R) (2018).

[3] J.O. Daldrop, B.G. Kowalik, R.G. Netz, Phys. Rev. $X$ 7, 041065 (2017).

[4] V. Lisý, J. Tóthová, Results Phys. 12, 1212 (2019).

[5] A.O. Caldeira, A.J. Legget, Ann. Phys. 149, 374 (1983).

[6] R. Zwanzig, Nonequilibrium Statistical Mechanics, Oxford University Press, New York 2001.

[7] V. Lisý, J. Tóthová, Transp. Theory Statist. Phys. 42, 1 (2013). 